\title{
Consecuencias de la infección malárica gestacional en la función inmune y en la inmunomodulación de la madre y el neonato
}

\author{
Catalina Álvarez-Larrotta y Jaime Carmona-Fonseca²
}

\section{Consequences of gestational malaria infection in the immune function and immunomodulation of mother and newborn}

Pregnancy-associated malaria is an understudied event in Latin America. Most works about malaria in pregnancy have been conducted in Africa. These studies indicate that the infection generates immune response modulation and alterations in the placental environment, key factors for the proper development of the fetus and neonate. Immunity against Plasmodium spp is complex since involves several factors that increase the possible infection outcomes. One of these immunological outcomes is the immune response modulation towards a regulatory profile, which is advantageous for the persistence of the parasite in the host; additionally, it could generate adverse events in the general immune response of infected individuals. The objective of this review is to address the Plasmodium spp mechanisms of modulation in the host immune response and expose the consequences of malarial infections in the mother-neonate context.

Keywords: Malaria; Plasmodium; inmunomodulation; gestation, neonate.

Palabras clave: Malaria, Plasmodium; inmunomodulación; gestación; neonato.

\section{Introducción}

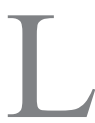

a infección plasmodial durante la gestación (malaria asociada al embarazo) puede causar malaria gestacional (infección de la madre), malaria placentaria (infección de la placenta) y malaria congénita (infección del neonato hasta los 28-30 días de edad) ${ }^{1}$. Estados Unidos de América, Brasil y Colombia son los países con mayor número de estudios. En Colombia en los últimos quince años se ha estudiado la malaria asociada al embarazo en la zona de Urabá Antioqueño-cuencas altas de los ríos Sinú y San Jorge en Córdoba-Bajo Cauca Antioqueño ${ }^{2}$. En esta región se encontró una frecuencia de infección en gestantes de $45 \%$ determinada a través de reacción de polimerasa en cadena (RPC) cuantitativa en tiempo real. La malaria placentaria es el evento que mayor frecuencia presenta, con 57\% de infección, seguida de malaria gestacional con $49 \%$ y la malaria congénita con $29 \% \%^{3}$; es clave decir que $79 \%$ de las infecciones correspondieron a infecciones submicroscópicas ${ }^{3}$. El historial de la malaria durante la gestación también se asoció con cambios histológicos en la placenta y bajo peso al nacer ${ }^{3}$. De igual manera, en esta población de mujeres se logró identificar en la placenta un aumento de infiltrado inflamatorio, de células en pre-apoptosis y apoptosis, además de la expresión de citoquinas pro-inflamatorias ${ }^{4}$.
En Colombia, los pocos estudios conocidos indican que el peso del neonato puede ser inferior o normal cuando se compara con el de los no expuestos a la infección durante la gestación ${ }^{5}$. En la región donde se han concentrado los estudios colombianos sobre malaria asociada al embarazo, la anemia materna es frecuente y más si sufren infección plasmodial en el embarazo ${ }^{5,6}$.

El embarazo aumenta la susceptibilidad a infecciones maláricas y los efectos de éstas van más allá de la infección gestacional, pues diversos autores informan que las consecuencias se extienden al neonato y a los primeros años de vida ${ }^{7,8}$. Al parecer, la exposición en útero a antígenos del parásito resulta en el estímulo de una respuesta inmune inadecuada para el normal desarrollo del lactante ${ }^{8}$. Actualmente, hay diversos estudios que asocian las infecciones maláricas con baja eficiencia en la respuesta inmune a otros tipos de infecciones, ${ }^{9}$ con tolerancia a la malaria ${ }^{10}$ e incluso con deficiencia en la respuesta a la vacunación ${ }^{11,12}$. La mayoría de los hallazgos asociados a la influencia de la malaria en la vacunación se centran en África, continente con alta transmisión de Plasmodium falciparum. Estos estudios muestran una disminución en las concentraciones plasmáticas de anticuerpos frente a vacunas como toxoide tetánico, toxoide diftérico, antígenos de Haemophilus influenzae tipo b o Neisseria meningitidis y en niños con exposición
'Estudiante de doctorado,

Corporación Ciencias Básicas Biomédicas, Grupo Salud y Comunidad, Universidad de Antioquia. Medellín, Colombia.

${ }^{2}$ Coordinador Grupo Salud y Comunidad, Docente e Investigador Facultad de Medicina, Universidad de Antioquia. Medellín, Colombia.

Los autores declaramos que no hay conflicto de intereses.

Recibido: (última versión): 20 de agosto de 2018

Aceptado: 5 de marzo de 2019

Correspondencia a: Catalina Álvarez-Larrotta catalinaalvarez195@gmail.com 
a antígenos del parásito durante la gestación (mujeres con malaria gestacional y placentaria microscópica $)^{13-15}$. Algo similar se ha encontrado en gestantes africanas con malaria frente al toxoide tetánico ${ }^{16} \mathrm{y}$, al parecer, también sucede en gestantes colombianas frente a ese toxoide ${ }^{17}$. Es necesario aclarar que los procesos de vacunación son influenciados por múltiples factores, como estado nutricional, deficiencia de vitamina $\mathrm{A}$, infección por parásitos intestinales, entre otros ${ }^{18}$.

En todos los países endémicos de malaria, gran parte de las infecciones maternas y placentarias son submicroscópicas ${ }^{3,19,20}$. Pese a conocerse la frecuencia y el impacto que estas infecciones generan en la salud pública, aún no se conoce la magnitud de sus consecuencias. La interacción de Plasmodium spp. con los componentes del sistema inmune es compleja e involucra diversos procesos que conducen a un rango amplio de respuestas. Estos procesos son, por ejemplo:

a) Variabilidad antigénica del parásito en cada uno de sus estadios durante la infección; b) Genotipo y fenotipo de adherencia de la cepa parasitaria infectante; c) Clase de antígeno (proteínas parasitarias, productos como hemozoína, glicosil fosfatidil inositol y vesículas extracelulares) reconocido por los diferentes receptores de las células presentadoras de antígeno; d) Tipo de receptor del sistema inmune que capta el antígeno (receptores tipo Toll (TLR siglas en inglés), receptores recolectores o basureros (scavenger), receptores de complemento, receptores Fc $\gamma$, entre otros. Los receptores Fc $\gamma$ permiten que células como linfocitos B (LB), células dendríticas, células asesinas naturales (natural killer), macrófagos, neutrófilos, entre otras, capten anticuerpos IgG adheridos a antígenos. La estimulación de cada de uno estos receptores, genera una señal intracelular que forma las características de la respuesta inmune.

La sinapsis inmunitaria o inmunológica es una estructura supramolecular transitoria y concertada, que tiene como propósito primario permitir un contacto estrecho de un linfocito con su célula objetivo ${ }^{21}$. Las imágenes de la interacción entre células inmunitarias muestran conexiones semejantes a las que entablan las neuronas para comunicarse. El estudio de estas sinapsis arroja luz sobre las redes de intercambio de información que las células tejen para cumplir sus funciones ${ }^{22}$. La complejidad de la sinapsis inmunológica entre linfocitos y células presentadoras de antígeno es variable, las combinaciones de los diferentes antígenos del parásito y los receptores ya descritos, puede conducir a malaria grave o a malaria asintomática. Además, no todas las interacciones de Plasmodium spp y sus productos con las células del sistema inmune resultan en activación de la respuesta efectora para combatir al patógeno, pues también se puede producir anergia (incapacidad de los linfocitos de reaccionar ante la presencia de un antígeno) o una respuesta reguladora.
Según diferentes hallazgos, las infecciones por Plasmodium spp promueven modulación de la respuesta inmune; sin embargo, cada componente del sistema inmune ha sido evaluado de manera individual. Este estudio estrictamente analítico o fragmentado resulta en una visión en un solo plano de la modulación. Plasmodium spp y sus productos (hemozoína, vesículas extracelulares, etc.) alteran la respuesta de células presentadoras de antígeno y conducen a un aumento de células reguladoras ${ }^{23,24}$. Estos sucesos generan un desequilibrio inmunológico que puede resultar en deficiencias en la respuesta inmune generada por linfocitos T (LT) ayudadores o helper.

La complejidad de esta infección parasitaria está controlada en gran medida por las características del ciclo de vida de Plasmodium spp., que incluye varios hospederos, en los cuales puede tener reproducción asexual o, en otros, sexual, con estadios diferentes en su composición y función antigénica. El ciclo sexual es desarrollado en el vector hembra Anopheles spp, el que se alimenta de sangre de individuos infectados tomando gametocitos macho y hembra circulantes. El ciclo esporogónico del parásito lleva a la formación de los esporozoítos y se desarrolla en el intestino de la hembra Anopheles spp, a partir de los gametocitos. El humano es infectado por los esporozoítos tras la picadura de la hembra Anopheles spp infectada; estos esporozoítos en el humano desarrollan el ciclo esquizogónico del parásito. En este ciclo, los esporozoítos que ingresan por inoculación cutánea migran hacía el hígado por vía hematógena o linfática hasta ingresar en un hepatocito y formar los esquizontes hepáticos (formas parasitarias compuestas por múltiples merozoítos). Las formas hepáticas, llamadas hipnozoítos, se desarrollan sólo en especies como $P$. vivax y $P$. ovale, entre las que infectan a humanos; los hipnozoítos son responsables de las recaídas. La generación de los esquizontes hepáticos dura aproximadamente 15 días y, luego de su formación, salen los merosomas a la circulación sanguínea, donde estallan y cada merozoíto se dispone a invadir un eritrocito. Dentro del eritrocito, el merozoíto madura a trofozoíto joven, y posteriormente, a trofozoíto maduro. Por último, el trofozoíto maduro se transforma a esquizonte circulante (compuesto por múltiples merozoítos), que estalla y cada merozoíto invade un nuevo eritrocito.

En esta fase sanguínea, algunos parásitos se transforman a gametocitos, que son formas extracelulares y que serán captados por las hembras Anopheles spp para iniciar nuevamente el ciclo.

El ciclo de vida de Plasmodium spp genera gran variedad de formas o estadios parasitarios tanto intracelulares como extracelulares. En las formas intracelulares se observa tropismo por diferentes tipos celulares, lo que refleja la variedad de proteínas propias del parásito involucradas en el período patogénico de la malaria. 


\section{Objetivo de la revisión}

Abordar los mecanismos por los cuales Plasmodium spp modula la respuesta inmune del hospedero y exponer las consecuencias de las infecciones maláricas en el contexto madre-neonato, con el fin de plantear propuestas de investigación que permitan definir la amplitud de las implicaciones de la malaria en la salud pública.

\section{Metodología}

Se realizó una búsqueda en las bases de literatura biomédica PubMed, Scopus y World of Science (WOS). Se emplearon varias estrategias de búsqueda usando términos MeSH y no MeSH. Se consultaron repositorios de universidades en América para buscar tesis de grado. El tema central de la búsqueda fue la influencia de las infecciones plasmodiales en la función inmune y en la inmunomodulación de la respuesta materna y neonatal. Esta no es una revisión sistemática. La búsqueda de información se hizo hasta julio de 2018.

\section{Aspectos inmunes en las infecciones con Plasmodium}

\section{Aspectos inmunes en las infecciones con estadios pre-eritrocitarios de Plasmodium spp}

Los esporozoítos son estadios móviles parasitarios que ingresan al hospedero humano por la picadura de la hembra Anopheles spp. Estas formas parasitarias son inyectadas en la piel, encontrándose en la dermis, epidermis e incluso en los folículos pilosos ${ }^{25}$. Los esporozoítos migran desde la piel mediante un movimiento de deslizamiento (gliding) hacia los vasos sanguíneos o linfáticos, cuyo alcance les permite llegar al hígado. En este órgano, invaden a los hepatocitos mediante la unión de la proteína de superficie circumsporozoítica (CSP) y el heparán sulfato, un glicosaminoglicano presente en la membrana de estas células. El esporozoíto puede atravesar varios hepatocitos hasta establecerse finalmente para formar su vacuola parasitófora, que genera el esquizonte hepático.

Durante el recorrido, los esporozoítos tienen contacto con las células del sistema inmune, tanto de la inmunidad innata como de la adaptativa. En el caso de inmunidad innata, se involucran el sistema de complemento y los TLR, entre otros receptores. En el caso de respuesta adaptativa se observa la participación de células $\mathrm{T}$ efectoras e inmunoglobulinas específicas de antígeno. Uno de los contactos descritos en inmunidad innata es mediante el reconocimiento de las moléculas de glicosil fosfatidil inositol contenidas en la membrana parasitaria por el TLR2. Esta interacción promueve la activación de la respuesta inmune con reconocimiento, fagocitosis, presentación antigénica y diferenciación de células T efectoras ${ }^{26,27}$. La CSP parece ser la proteína presentada con más frecuencia por el complejo mayor de histocompatibilidad (CMH) en estadios preeritrocitarios. Esta puede generar títulos detectables de anticuerpos específicos y es una proteína diana en el diseño de vacunas antimalaria.

En los individuos con historia previa de malaria y seropositivos para la CSP, la respuesta inmune se inicia con la opsonización de los esporozoítos por anticuerpos IgG específicos, la captación de los complejos por las células presentadoras de antígeno mediante receptores Fcy, activación y amplificación de células T efectoras y de células $\mathrm{B}$ con maduración de afinidad de los anticuerpos ${ }^{28}$. Los anticuerpos anti-CSP neutralizan al esporozoíto de manera independiente de la subclase de inmunoglobulina y participan en los procesos de opsonización junto con el complemento, lo que favorece el control de la infección ${ }^{29,30}$. Estos anticuerpos anti-CSP son detectables en el suero de individuos sanos y residentes de zonas endémicas, en quienes además, se han detectado células B de memoria específicas para CSP de P. falciparum $^{28}$.

Una vez que el esporozoíto se encuentra en el hígado, se despliega otro mecanismo de respuesta inmune, como es la fagocitosis por las células de Kupffer o de BrowiczKupffer. Se pueden diferenciar células efectoras del tipo $\mathrm{LT} \mathrm{CD} 8^{+}$que reconocen epítopos del parasito mediante el CMH clase I en la superficie de los hepatocitos infectados. Estas células efectoras promueven la lisis de los hepatocitos mediante perforinas, FasL, granzimas y la producción del interferón gamma (IFN $\gamma$ ) y el factor de necrosis tumoral (FNT) ${ }^{25}$. Mediante el reto in vitro de células dendríticas con P. berghei (plasmodio de roedores), se demostró la inducción de LT citotóxicos CD8+ específicos contra la proteína CSP y la posterior producción de IFN $\gamma$ e inducción de una respuesta inmune tipo Th $1^{31}$.

\section{Aspectos inmunes en las infecciones con estadios eritrocitarios de Plasmodium spp}

Igual que en estadios pre-eritrocitarios, las células presentadoras de antígeno pueden tener un primer contacto con los parásitos mediante respuesta inmune innata o adquirida $^{32}$. Las moléculas asociadas a estadios eritrocitarios reconocidos por diferentes receptores de la respuesta inmune innata son: a) glicosil fosfatidil inositol, producto de la ruptura del eritrocito parasitado, que promueve el estímulo en células presentadoras de antígeno a través del TLR2 $2^{33}$; b) hemozoína, producto de degradación de la hemoglobina, reconocida por el TLR9 $9^{34}$; c) el complejo ADN-hemozoína de $P$. falciparum, reconocido por el TLR9. ${ }^{35}$ Cada uno de estos receptores es prescindible en la generación de la respuesta inmune ${ }^{36}$. La Figura 1 esquematiza estos procesos de reconocimiento.

Las diferentes moléculas de Plasmodium spp reconocidas por los TLR promueven una respuesta inmune 
a)

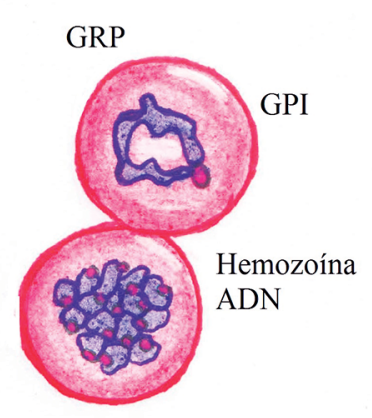

c)

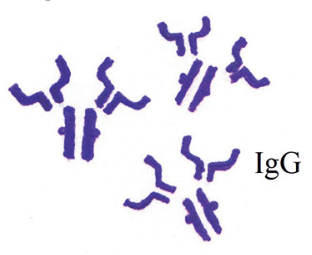

b)

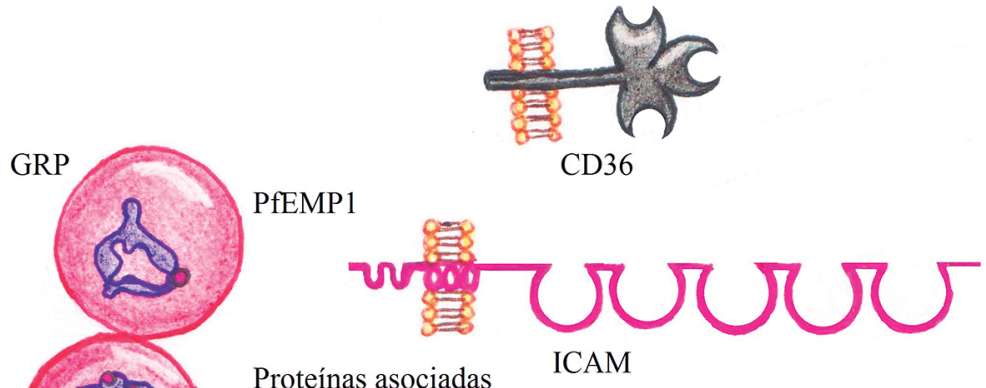

a genes vir

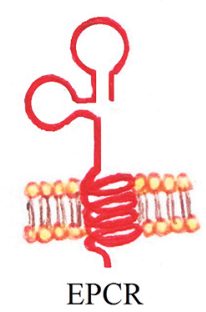

Figura 1. Diferentes mecanismos de interacción del eritrocito parasitado (GRP) con las células presentadoras de antígeno (CPA). a) Los receptores TLR2 y TLR9 de la respuesta inmune innata interactúan con el glicosilfosfatidilinositol (GPI) y la hemozoína más ADN respectivamente. b) Los GRP expresan es su superficie proteínas especificas del parasito que tienen diferentes fenotipos de adhesión a varios receptores, dentro de estos, CD36, ICAM y el receptor endotelial de la proteína C reactiva (EPCR). c) Adicionalmente, puede interactuar con los receptores Fcy mediante los anticuerpos lgG específicos contra Plasmodium spp y con los receptores CR1 y CR2 a través de la fracción C3b del complemento.

inflamatoria Th1, la que es mantenida principalmente por IFN $\gamma$. Esta respuesta se caracteriza por la producción de FNT, las interleuquinas (IL) $12,1 \beta$ y 6 y por aumento en la expresión de $\mathrm{CMH} \mathrm{II}{ }^{37}$. El IFN $\gamma$ induce aumento de la producción de especies reactivas de oxígeno y de óxido nítrico $(\mathrm{ON})$ en las células fagocíticas, lo que lleva a la eliminación de los eritrocitos infectados (Ei) y favorece la presentación antigénica ${ }^{38}$. El IFN $\gamma$ promueve un aumento de la población de linfocitos efectores Th1 que apoyan su producción y amplifican la respuesta; además, la IL-12 estimula la producción de IFN $\gamma$ en los LT, lo que fomenta un perfil Th1 ${ }^{39}$.

Los niveles de ON producido por las células fagocíticas se incrementan a medida que aumenta la parasitemia, consecuente con la respuesta Th1. Sin embargo, durante el proceso de regulación de la respuesta se producen citoquinas anti-inflamatorias como la IL-10 que previenen el daño tisular. Tras la captación de los antígenos y su presentación a través de las células presentadoras de antígeno en los órganos linfoides, se fomenta la diferenciación de LB específicos contra antígenos de Plasmodium spp. La diferenciación de la población celular B supone el desarrollo de la respuesta humoral. Las inmunoglobulinas (Ig) $\mathrm{G} 1$ e $\operatorname{IgG}^{40}$ son las más frecuentes y producidas en mayores cantidades tras la respuesta inmune a este patógeno; ellas se caracterizan por tener una alta capacidad de fijación de complemento, por presentar facilidad de unión a receptores Fc $\gamma$ de células presentadoras de antígeno y además pueden realizar traspaso placentario (Figura 2) ${ }^{41}$.

El desarrollo de la respuesta inmune contra los estadios eritrocitarios de Plasmodium spp tiene dos aspectos importantes. El primero es la intracelularidad del parásito, lo que le confiere la capacidad de evadir varios mecanismos de la respuesta inmune innata. El segundo es la gran variabilidad antigénica asociada a estas formas parasitarias (trofozoítos jóvenes, trofozoítos maduros y esquizontes). La capacidad de variabilidad funciona como un mecanismo de escape a la respuesta inmune adquirida.

\section{Mecanismos de inmunomodulación propuestos en infección por Plasmodium spp}

La inmunomodulación se genera por la acción de diversos componentes, cuya naturaleza puede ser proteica, lipídica o de ácidos nucleicos propios del parásito. Estos componentes intervienen sobre los procesos de 


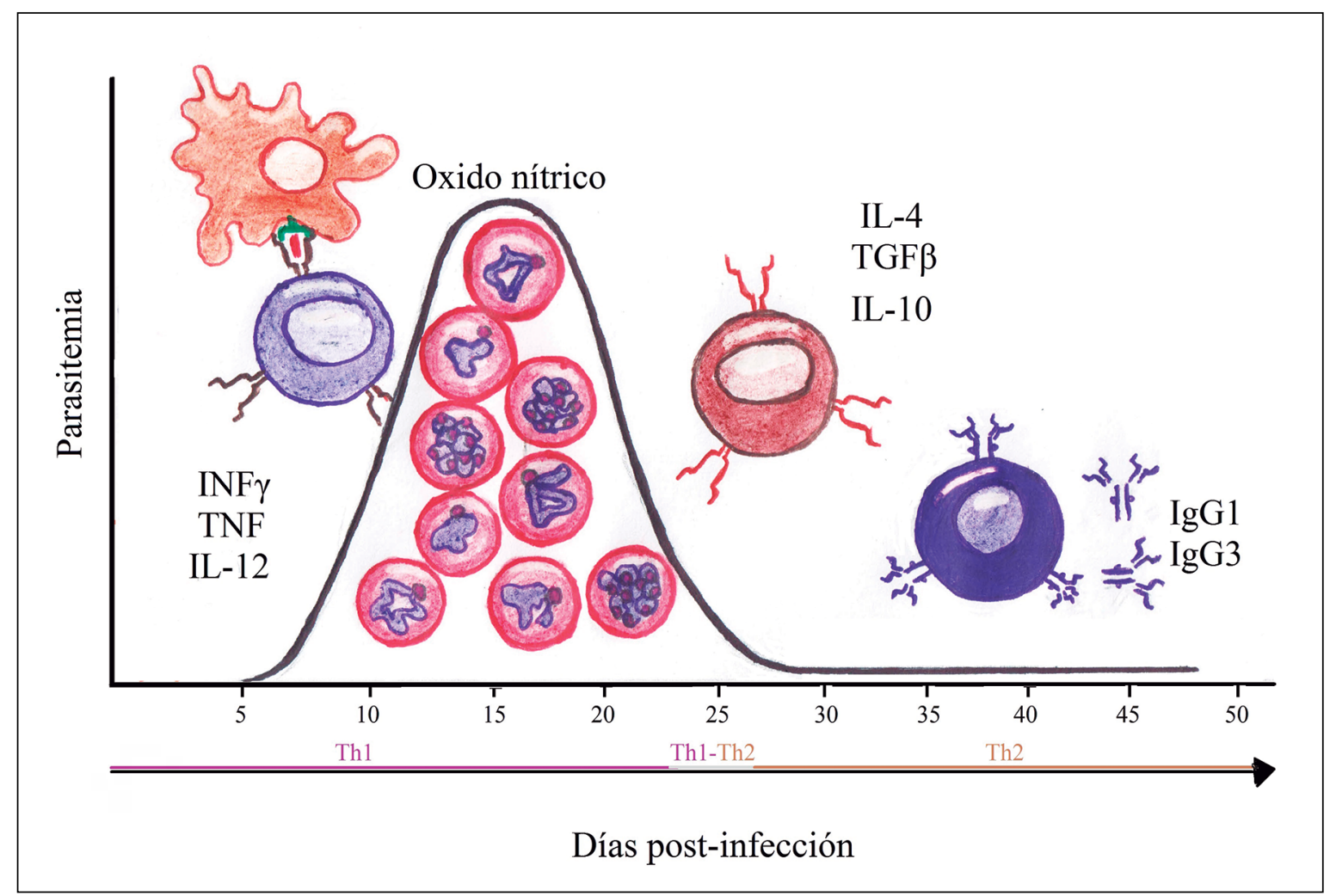

Figura 2. Cinética de la respuesta inmune contra estadios asexuales sanguíneos de Plasmodium spp. Las células presentadoras de antígeno realizan el primer contacto con el patógeno y producen citoquinas pro-inflamatorias que promueven la respuesta Th1 y la migración y activación de macrófagos que producen óxido nítrico para el control de la infección. Posteriormente se produce un aumento de citoquinas anti-inflamatorias para controlar la respuesta y se induce la respuesta tipo Th2 caracterizada por la IL-4 y la siguiente diferenciación de LB y producción de anticuerpos específicos. autorregulación habituales que dirigen el sistema de defensa inmunitario y pueden contribuir, tanto a los procesos fisiopatológicos de la enfermedad, como a la evasión de la respuesta inmune. Por tal razón, los llamados compuestos realizan estímulos en diferentes receptores de diversas células, principalmente, las células inmunes y alteran la respuesta habitual contra el patógeno. Una célula del sistema inmune puede tener varios estímulos simultáneos y tiene dos opciones de activación. La primera es la activación hacia un perfil pro-inflamatorio y la segunda es la activación hacia un perfil anti-inflamatorio o regulador.

\section{Interacción de Plasmodium spp con monocitos, macrófagos y células dendríticas}

Los monocitos, macrófagos y células dendríticas, en su papel de células presentadoras de antígeno, interactúan con los eritrocitos parasitados de dos maneras: directa (contacto directo con ellos) o de forma indirecta (contacto con productos del parásito). Las células presentadoras de antígeno tienen diferentes receptores que median la interacción: unos se unen a distintas moléculas expresadas en los eritrocitos parasitados y otros a productos del parásito. Cada forma de interacción, ya sea de manera dependiente o independiente de contacto, promueve un estímulo y una función efectora en la célula inmune ${ }^{42}$.

La interacción de las células presentadoras de antígeno con Plasmodium spp en reconocimiento típico de patógenos dentro de la respuesta inmune se puede realizar por varias vías (Figura 1a):

- por la vía del complemento por medio de la proteína $\mathrm{C} 3 \mathrm{~b}$, la que opsoniza al patógeno y promueve su fagocitosis por los receptores CR1 (receptor $1 \mathrm{del}$ complemento), sinónimo de CD35;

- por opsonización con anticuerpos específicos, cuando hay exposición previa al parásito; en este contexto se promueve la fagocitosis del patógeno mediante los receptores Fc $\gamma$;

- mediante los TLRs, por medio de moléculas como glicosil fosfatidil inositol y hemozoína, se promueve fagocitosis ${ }^{42}$.

La captación y fagocitosis de los eritrocitos infectados es una vía importante en la eliminación parasitaria. La capacidad para la fagocitosis es diferente en cada individuo y puede ser facilitada por el clon parasitario infectante ${ }^{43}$. Sin embargo, también depende del receptor que realice la captación del parásito. En todos estos casos se promueve una respuesta pro-inflamatoria que contribuye a combatir la infección. 
En otro contexto, existen interacciones no típicas mediadas por la capacidad de adhesión del parásito a diferentes moléculas presentes en distintos tipos celulares. En este sentido, el parasito puede ser captado a través de receptores recolectores o recicladores de desechos (scavenger) por ser un patógeno intracelular. Estos receptores reconocen y captan cuerpos apoptóticos e inducen la inhibición de la función de las células presentadoras de antígeno para evitar el procesamiento de antígenos propios. Además, el receptor recolector CD36 es una molécula presente en fagocitos y células asesinas naturales (células NK) y es un blanco de anclaje frecuente de Plasmodium spp, ya que proteínas producidas por el parásito tienen afinidad por este receptor (Figura 1b) ${ }^{44}$. Por lo tanto, cuando el parásito interactúa con las células presentadoras de antígeno mediante este receptor, se genera una respuesta inmune anti-inflamatoria favoreciendo la viabilidad el parásito.

La característica de adhesión propia del parásito define el perfil de la respuesta que se desarrollará. De esta manera, la modulación de la respuesta inmune se fundamenta en el fenotipo de adhesión del parásito, el tipo de receptor al que se adhiere, a la afinidad de unión con el receptor y a la cantidad de estímulos que recibe cada célula (amplificación de la respuesta). En este sentido, la unión con el receptor CD36 está asociada a casos de malaria no complicada, debido a que la señalización intracelular generada tras la activación de este receptor es una respuesta anti-inflamatoria que regula el reciclaje de las moléculas que capta ${ }^{45}$, mientras que la unión al receptor ICAM (intercellular adhesion molecule 1) o CD54 se asocia a casos de malaria complicada, porque la señalización intracelular mediada por este receptor conduce a una respuesta pro-inflamatoria. Recientemente, se describió la unión al receptor EPCR (endothelial protein C receptor) expresado por el endotelio; tras esta unión, se inhibe la activación de la proteína $\mathrm{C}$ reactiva (PCR), con actividad anti-inflamatoria, anti-trombótica y actividad protectora endotelial ayudando a mantener la integridad vascular. Este tipo de unión se ha descrito en casos de malaria cerebral y malaria complicada (Figura $1 b)^{43,45,46}$.

Además, el secuestro de los eritrocitos parasitados en diferentes órganos y la formación de rosetas ayudan a la fisiopatología de la enfermedad. La formación de estas estructuras está directamente asociada a la producción de proteínas con propiedades de adhesión típicas del parásito y su exportación a la membrana del eritrocito parasitado. Las rosetas facilitan la invasión de los eritrocitos adyacentes, dificultan el reconocimiento de los eritrocitos infectados por las células del sistema inmune y pueden generar obstrucción vascular ${ }^{45,46}$. Por lo tanto, hay una relación directa entre el fenotipo adherente del parásito y la respuesta inmune inducida en las células presentadoras de antígeno ${ }^{44}$. Además, las diferentes vías de fagocitosis modulan de manera variable la producción de citoquinas; por esta razón, se observa gran variedad de fenotipos clínicos de la enfermedad, encontrando desde casos de malaria complicada hasta infecciones asintomáticas.

Se ha planteado que la función de las células presentadoras de antígeno se puede ver reducida en las infecciones maláricas. La hemozoína no siempre activa a las células presentadoras de antígeno; la captación e ingreso de eritrocitos parasitados con contenido de hemozoína al macrófago, inhibe la fagocitosis y altera la formación del fagolisosoma por disminución en la generación de radicales libres de oxígeno y nitrógeno ${ }^{47}$. Esta disfunción en los macrófagos afecta la respuesta del individuo y favorece la supervivencia del parásito; adicionalmente, podría explicar la frecuente asociación de las infecciones maláricas con bacteriemia en niños ${ }^{48,49}$.

Las células dendríticas son las células presentadoras de antígeno "profesionales"; por esta razón, tienen el papel importante en la resolución de los procesos infecciosos. En células dendríticas expuestas in vitro a eritrocitos parasitados se observó disminución de la expresión de $\mathrm{CMH}$ clase II, moléculas de adhesión y coestimuladoras y, adicionalmente, una reducción en la respuesta a estímulos posteriores con lipopolisacarido ${ }^{50}$. Se describió que las diferencias en los estímulos (tipo de receptor y afinidad de interacción) pueden generar, tanto la activación de las células dendríticas como la inhibición de sus funciones. Por otra parte, hay pruebas de que las formas asexuales de $P$. berghei, $P$. chabaudi (plasmodio de roedores) y $P$. yoelii (plasmodio de roedores) tienen tropismo por las células presentadoras de antígeno de murinos. Por esta razón, los parásitos pueden invadir a las células presentadoras de antígeno y asegurar un lugar inmunoprivilegiado en el hospedero con la opción de llegar incluso a bazo y médula ósea ${ }^{51,52}$.

En síntesis, la participación de las células presentadoras de antígeno en la respuesta a Plasmodium spp es importante y abarca un amplio espectro de desenlaces. La variación no sólo depende del tipo de contacto con la célula sino que también depende de las características fenotípicas del parásito para mediar esta interacción.

\section{Equilibrio linfocitos $T$ reguladores versus linfocitos T efectores}

La respuesta inmune a infección por Plasmodium spp se ha descrito como de baja efectividad ${ }^{53}$, es decir, de baja capacidad para controlar y eliminar la infección. Varios factores dificultan el desarrollo de la respuesta inmune y de la generación de una adecuada inmunidad adquirida. Dentro de estos factores se encuentran los inherentes a la biología del parásito, que dificultan el desarrollo de un estándar inmunogénico; esto se evidencia en la variación de la presentación clínica de la enfermedad alrededor del mundo. Estos factores inherentes a la biología del parásito 


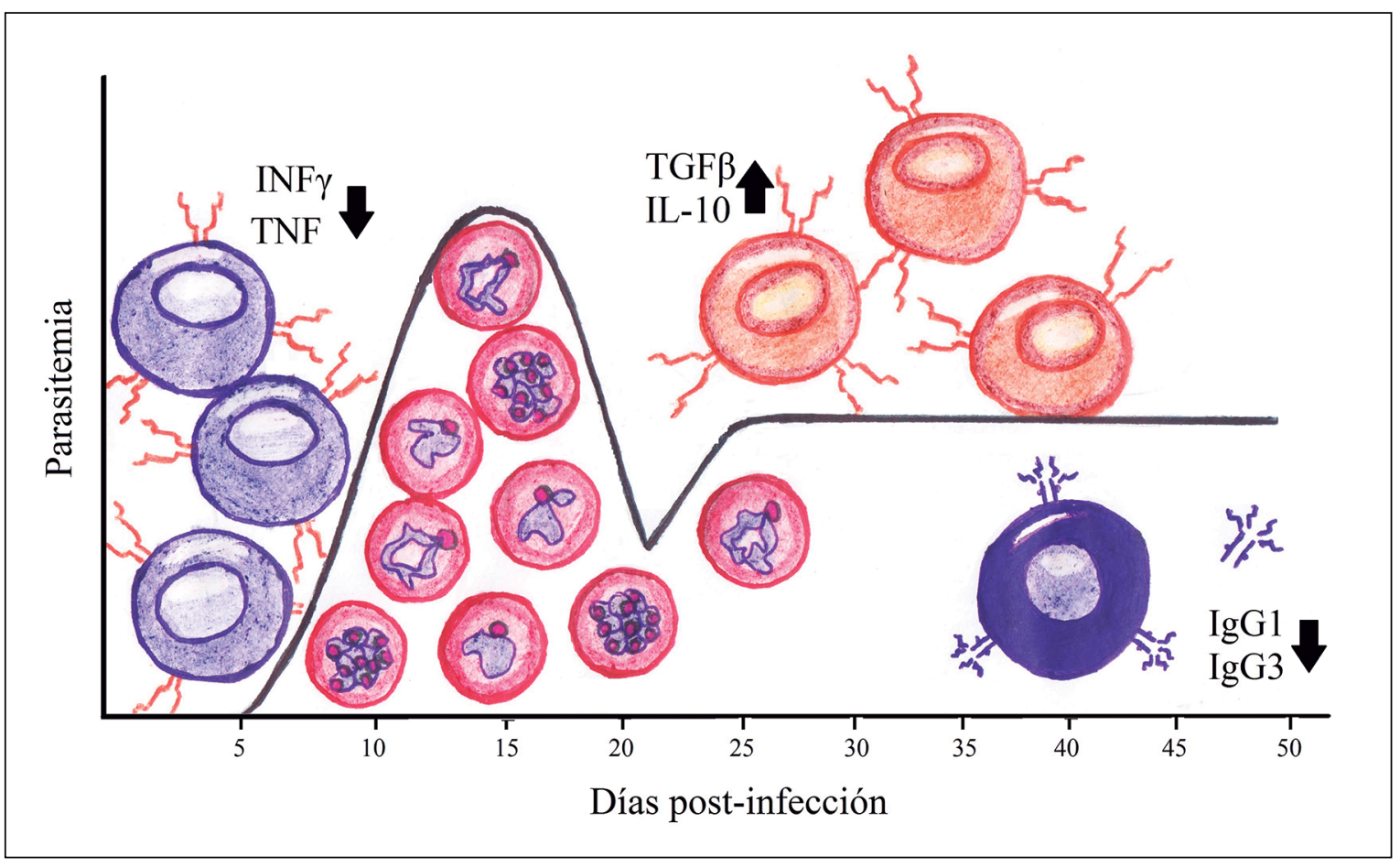

Figura 3. Figura 3. Efecto de la activación no especifica de los linfocitos $T$ reguladores en infección por Plasmodium chabaudi. En ratones infectados con P. chabaudi se observa aumento de las células $T$ reguladoras; estas células controlan la respuesta Th1 antes del aclaramiento parasitario y dificultan la generación de la respuesta Th2. Este aumento de células reguladoras dificulta el control de la infección y el desarrollo de una eficiente respuesta de inmunidad adquirida y producción de IgG. corresponden a la capacidad de variación antigénica de Plasmodium spp y la variedad de formas parasitarias que componen su ciclo de vida en el hospedero humano.

En modelos murinos infectados con $P$. chabaudi se encontró disminución de IFN $\gamma$, FNT e IL-12 antes del control de la parasitemia periférica ${ }^{54}$. Estos hallazgos sugirieron la importancia del papel de los LT reguladores, mediadores de los LT efectores en las respuestas inflamatorias y aclaran su implicación en la transición de respuesta Th1 a Th2 (Figura 3$)^{55}$.

Los LT reguladores son inducidos principalmente por IL-10 y factor de crecimiento transformante $\beta$ (TGF $\beta$ ) y su proliferación y supervivencia es dependiente de la producción de IL-2 realizada por LT CD4 $4^{+}$. IL-2 actúa como molécula autorreguladora por medio de $\mathrm{CD} 25^{+}$ (cadena $\alpha$ del receptor de IL2). La acción inmunosupresora de los $\mathrm{T}$ reguladores contempla la producción de citoquinas anti-inflamatorias, como TGF $\beta$, IL-4 e IL-10, y la inducción de apoptosis en células T efectoras de manera no específica ${ }^{56,57}$. Se han descrito dos poblaciones de LT reguladores: los naturales diferenciados en el timo y los inducidos generados en la periferia a partir de $\mathrm{LT} \mathrm{CD}^{+}$ por estímulos de citoquinas ${ }^{57}$.

Hay evidencia del aumento de la población de T reguladoras en murinos y humanos con infección malárica. En ratones infectados con $P$. yoelii, $P$. berghei y $P$. chabaudi se observó que el número de LT reguladores es proporcional a la carga parasitaria. Esta proporcionalidad se vio en los casos de malaria grave, mientras que en los casos de malaria no complicada no se conservó ${ }^{58}$. En ratones infectados con $P$. yoelii se encontró una mayor producción de IL-10 en la población de LT reguladores inducidos que en la población de LT reguladores naturales ${ }^{23}$. Es clara la diferenciación de LT reguladores en infecciones maláricas, pero es discutido el sentido en el que pueden irrumpir en el desarrollo de una respuesta inmune adecuada. En ratones, se asocia una temprana diferenciación de LT reguladores con una deficiente inmunidad adquirida por alteración de la expansión de células efectoras. Los cambios en los niveles relativos de LT reguladores y LT efectores generan más información respecto a la afectación del sistema inmune y su homeostasis. En el mismo modelo experimental, se indicó que cuando se presenta una inducción temprana de LT reguladores, disminuye la razón entre $\mathrm{LT}$ reguladores y LT efectores y se genera una potencial inmunosupresión, por predominio de la respuesta reguladora ${ }^{59}$.

In vitro, $P$. falciparum induce diferenciación de LT reguladores, pero IL-10 y TGF $\beta$ son indispensables para su generación. En ratones infectados con P. falciparum se encontró un aumento en la población de LT reguladores, apoyando que la infección con Plasmodium spp diferencia a LT reguladores ${ }^{24}$. En adición, tanto en individuos con malaria asintomática como grave, hay mayor expresión del receptor del FNT celular tipo II en la población de LT reguladores. El receptor del FNT celular tipo II es un 
marcador de la actividad inmunosupresora de LT reguladores, importante en el control de la proliferación de LT efectores, ya que es un receptor asociado a apoptosis ${ }^{60}$.

Se propuso que antígenos y factores solubles de Plasmodium spp inducen la diferenciación de LT reguladores, lo que indica un mecanismo de inducción adicional a las moléculas diferenciadoras y de proliferación: IL-2, IL-10 y TGF $\beta$. Se plantea que Plasmodium spp puede inducir LT reguladores mediante los TLRs. En ensayos in vitro, hay inducción de T reguladoras mediante TLR9, el cual está involucrado en el reconocimiento antigénico a Plasmodium spp. ${ }^{61}$.

\section{Las vesículas extracelulares en la inmunomodulación durante la infección por Plasmodium spp}

Las causas del desequilibrio en la respuesta inmune ante infecciones plasmodiales aún no son claras. Se han asociado antígenos y productos parasitarios que conducen a la activación inespecífica de células, expresión de citoquinas y receptores. Se han implicado los factores solubles producidos y exportados por el parásito fuera del eritrocito. Más recientemente, el nuevo blanco de estudio en el campo de la modulación inmune ha sido la evidencia de la producción de vesículas extracelulares por parte de Plasmodium spp y otros protozoos ${ }^{62}$.

El concepto vesículas extracelulares (VE) comprende las microvesículas y los exosomas, que se diferencian en tamaño y procedencia. Las VE son definidas como comunicadores celulares que pueden contener diversos productos (lípidos, péptidos, proteínas, microARN y ARN de interferencia $)^{63}$. Las microvesículas (100 a $\left.1000 \mathrm{~nm}\right)$ transportan moléculas de señalización y se comportan como transportadores transmembranales y, en malaria, su contenido genera la activación del complemento por las vías clásica y alternativa ${ }^{63}$. Complementario a lo anterior, causan auto-tolerancia en el hospedero, inmunosupresión y mimetismo molecular entre polipéptidos de antígenos parasitarios y del hospedero ${ }^{63}$. Los exosomas (30 a 100 $\mathrm{nm})$ son formados por endocitosis de segmentos de la membrana plasmática. Los eritrocitos infectados con $P$. falciparum liberan estas estructuras y se postula que ellas transfieren y propagan información que resulta ventajosa para el crecimiento de la población parasitaria en condiciones de estrés celular. También se ha demostrado que los exosomas promueven la diferenciación de las formas sexuales como un mecanismo de escape hacia el vector en respuesta a las condiciones adversas en el hospedero ${ }^{64,65}$.

Se han descrito múltiples interacciones de las vesículas extracelulares de Plasmodium spp con las células del hospedero. En modelos murinos infectados con $P$. berghei se demostró que las microvesículas derivadas de los eritrocitos infectados tienen la capacidad de inducir inflamación, principalmente por interacción con los ma- crófagos mediante TLR y contribuyen a la patogénesis ${ }^{66}$. La producción de las VE se asocia a estadios asexuales del parásito. Éstas contienen proteínas típicas de vesículas transportadoras propias del eritrocitos y proteínas del parásito. Se demostró que las microvesículas son inmunoestimuladoras e inducen una respuesta inflamatoria en los monocitos y un aumento en la quimiotaxis de neutrófilos; además, actúan como mensajeras entre los eritrocitos infectados mediando la diferenciación de formas sexuales del parásito por medio de microARN. Estos pequeños ARN pueden promover la transcripción de proteínas del parásito e incluso bloquear la producción de proteínas del hospedero, afectando la biología de las células ${ }^{67}$. Las microvesículas llevan a cabo su función induciendo su fagocitosis por las células presentadoras de antígeno o por fusión de membranas facilitada por la presencia de proteínas propias del hospedero ${ }^{63}$.

También hay concentraciones plasmáticas elevadas de microvesículas derivadas de eritrocitos y plaquetas en pacientes con infecciones maláricas por $P$. falciparum y $P$. vivax con diferente presentación clínica ${ }^{68}$. Además, al comparar las concentraciones de vesículas extracelulares provenientes de eritrocitos infectados con P. falciparum, $P$. vivax y $P$. malariae, se encontraron mayores concentraciones en los pacientes con malaria grave por $P$. falciparum $^{68}$. Hay evidencias que indican la interacción de las vesículas extracelulares con las células del sistema inmune; sin embargo, no son claras las consecuencias de estas interacciones. Teniendo en cuenta la irregularidad de la respuesta inmune contra Plasmodium spp y las características y gran variedad de contenidos de las vesículas extracelulares, éstas podrían estar involucradas en el desenlace clínico de las infecciones.

\section{Consecuencias de la malaria asociada al embarazo en el desarrollo inmune del neonato}

\section{Tolerancia inmune en niños hijos de madres con malaria placentaria}

La exposición temprana en útero a antígenos del parásito podría resultar en tolerancia inmune en los recién nacidos de mujeres con malaria placentaria. Estudios señalan que estos niños presentan mayor riesgo de infección malárica y que esta vulnerabilidad podría persistir durante años ${ }^{69}$. Los modelos murinos apoyan este planteamiento. En ratones recién nacidos se confirmó que la exposición a LT diferenciados por la CSP de $P$. falciparum conduce a tolerancia inmune en las crías ${ }^{70}$. Además, el paso de anticuerpos específicos contra antígenos de $P$. falciparum a las crías conlleva a una deficiente producción de anticuerpos en respuesta a los antígenos para los cuales habían adquirido inmunidad pasiva por traspaso vertical ${ }^{71}$. En 
humanos, la exposición a antígenos del parásito conduce a una sensibilización prenatal y genera niños con fenotipos tolerantes a infecciones por Plasmodium spp $^{71}$. Los niños son quienes presentan mayor morbimortalidad por esta enfermedad en zonas de alta endemia ${ }^{72}$.

La alteración en la inmunidad de los niños es apoyada por un aumento en la frecuencia de LT reguladores en sangre del cordón umbilical de los recién nacidos en zonas endémicas de malaria ${ }^{73}$. En Kenia, se describió que un subconjunto de niños expuestos a malaria placentaria puede adquirir un fenotipo tolerante a los antígenos eritrocitarios del parásito. Esta tolerancia puede persistir en la infancia y está asociada a una mayor susceptibilidad a la infección de la malaria y la anemia ${ }^{16}$. En la generación de tolerancia inmune en los neonatos no sólo ha sido asociada la proliferación de LT reguladores. También se ha asociado el paso de LT y LB activos y de anticuerpos específicos contra Plasmodium spp vía cordón umbilical ${ }^{10}$. Sin embargo, este fenómeno de tolerancia inmune en los neonatos aún es objeto de investigación.

En este campo, los resultados obtenidos aún son contradictorios. En un estudio en Camerún no se encontraron diferencias durante los dos primeros años de vida en la frecuencia de la malaria entre los nacidos de madres con malaria placentaria y los nacidos de madres sanas ${ }^{74}$. En otros estudios recientes, los resultados obtenidos sugieren que los niños nacidos de madres con malaria placentaria presentan un alto riesgo de adquirir mayor número de infecciones por $P$. falciparum en los dos primeros años de vida, en comparación con los nacidos de mujeres sin malaria placentaria ${ }^{75}$. A pesar de las discrepancias, la hipótesis de que la malaria gestacional puede generar efectos prolongados sobre la inmunidad y susceptibilidad a la malaria en los hijos, parece ser acertada ${ }^{76,77}$.

\section{Infecciones maláricas y la inmunomodulación en la respuesta a antígenos vacunales}

Un hallazgo con gran impacto en la salud pública de países endémicos de malaria es la alteración de la respuesta inmune a vacunas administradas a mujeres con malaria gestacional y placentaria y a sus neonatos ${ }^{13}$. Esto ha sido demostrado en niños vacunados con polisacárido capsular de Haemophilus influenzae tipo b y toxoide tetánico ${ }^{13}$. Más aún, en Papua, Nueva Guinea, aproximadamente $10 \%$ de los neonatos de madres con malaria placentaria por $P$. falciparum pueden no adquirir pasivamente una concentración plasmática protectora de anticuerpos contra el tétanos, inclusive en concentraciones adecuadas de anticuerpos maternos ${ }^{78}$. Asímismo, en Kenia se observó que las concentraciones de anticuerpos contra tétanos se redujeron en $48 \%$ en los recién nacidos cuyas madres tenían malaria placentaria crónica activa o pasada ${ }^{79}$ y en África Sub-sahariana la malaria placentaria se asoció con la respuesta inmune reducida 12 meses después del desafío inmunológico con BCG en bebés aparentemente sanos al nacer ${ }^{14}$.

Existen diversos factores que pueden llevar a deficiencias en la respuesta inmune protectora frente a la administración de vacunas. La desnutrición y las coinfecciones con parásitos intestinales inducen reducción en la calidad de la respuesta inmune, razón por la que, la malaria podría estar implicada pero no sería el único factor explicativo. Se ha descrito que la respuesta inmunogénica a la vacuna meningocócica disminuye en los niños bajo dos años de edad, principalmente en África Sub-sahariana cuando co-existen las infecciones por malaria y $\mathrm{VIH}^{12}$. Estos fenómenos podrían explicar la existencia de una reducida eficacia de las vacunas utilizadas en la inmunización infantil de rutina en comparación con los países no endémicos ${ }^{12}$.

Varios estudios demostraron la marcada diferencia en la eficacia de los inmunógenos en niños hijos de mujeres con y sin malaria gestacional en Gambia ${ }^{80}$. Estas observaciones aplican para la vacuna BCG administrada a los recién nacidos en el primer día de vida, probablemente, por un mecanismo de baja actividad de los LT. Adicionalmente, se realizaron estudios que encontraron bajas concentraciones plasmáticas en la producción de IFN $\gamma$ en adolescentes de Malawi en comparación a británicos inmunizados con esta misma vacuna ${ }^{81}$.

En este sentido, las medidas de intervención como la terapia intermitente preventiva (TIP) podrían tener efecto sobre la adquisición de inmunidad a antígenos vacunales. En Nigeria, la TIP con cloroquina resultó en mayores títulos de anticuerpos específicos contra $N$. meningitidis. Estos resultados sugieren que la respuesta inmune contra vacunas de antígenos bacterianos se puede ver afectada, tanto por malaria aguda como por malaria asintomática, por lo que, la administración de TIP en zonas endémicas podría contribuir a mejorar la respuesta a vacunas ${ }^{15}$.

\section{Conclusión}

Existen evidencias en modelos murinos e in vitro que apoyan la modulación de la respuesta inmune ejercida por Plasmodium spp. Además, estudios realizados en población afectada cuantifican y describen cambios en las poblaciones celulares y moléculas del sistema inmune en respuesta a la malaria. La mayoría de los estudios se han hecho en África una zona de alta y estable transmisión de la malaria. En América, una zona de inestable transmisión, se observan amplios patrones de respuesta a la infección, hallándose desde individuos asintomáticos hasta individuos con malaria grave. Los reportes realizados hasta el momento acerca de la modulación de la respuesta inmune en infección por Plasmodium spp evidencian su importancia. Estas infecciones están asociadas con procesos que generan alto impacto en 
la salud pública, como son la inmunización en madres gestantes y neonatos y la tolerancia inmune a la malaria en los neonatos. Esto debe ser evaluado en el contexto de la alta frecuencia de infecciones sub-microscópicas hallada en zonas endémicas ${ }^{4}$. Las evidencias obtenidas hasta el momento proporcionan bases para el conocimiento de los procesos de modulación inmune; sin embargo, es importante indagar sobre los mecanismos de desarrollo de la modulación para plantear alternativas que ayuden a disminuir el impacto del amplio espectro de las infecciones maláricas.

\section{Necesidades de investigación}

Dada la presencia de infecciones asintomáticas y sub-microscópicas en nuestro medio latinoamericano, específicamente la llamada cuenca de la Amazonía, hace falta explorar la presencia de inmunomodulación en las mujeres gestantes y neonatos con infecciones maláricas y su influencia en la respuesta inmune a malaria, antígenos vacunales y la co-infección con diferentes patógenos a largo plazo, así como, los mecanismos y la identificación de las moléculas generadas por Plasmodium spp involucradas en los procesos de inmunomodulación. Los resultados serían de gran importancia en el diseño de los planes de inmunización y podrían justificar cambios en el tratamiento antimalárico de las poblaciones gestantes expuestas a la infección.

\section{Resumen}

La malaria asociada al embarazo es un evento poco estudiado en América Latina. Los abundantes trabajos sobre el problema en África llevan a pensar que esta infección genera una modulación de la respuesta inmune y alteraciones en el ambiente placentario, eventos cruciales para el adecuado desarrollo del feto y el neonato. La inmunidad contra Plasmodium spp es compleja porque involucra diversos factores que amplían las posibilidades de desenlaces, los que finalmente conducen a los diferentes fenotipos clínicos de la enfermedad. Uno de los desenlaces inmunológicos en infecciones por Plasmodium spp es la modulación de la respuesta inmune hacía un perfil regulador. Esta regulación inducida por la infección malárica resulta ventajosa para la persistencia del parásito en el hospedero, y adicionalmente, podría generar eventos adversos en la respuesta inmune general de los individuos infectados. El objetivo de esta revisión es abordar los mecanismos con los cuales Plasmodium spp modula la respuesta inmune del hospedero y exponer las consecuencias de las infecciones maláricas en el contexto madre-neonato.

\section{Referencias bibliográficas}

1.- Carmona-Fonseca J, Maestre A. Incidencia de las malarias gestacional, congénita y placentaria en Urabá (Antioquia, Colombia), 2005-2007. Revista Colombiana de Obstetricia y Ginecología 2009; 60: 19-33. http://www. scielo.org.co/pdf/rcog/v60n1/v60n1a05.pdf.

2.- Carmona-Fonseca J. La Región "Urabá Antioqueño-Cuencas altas de los ríos Sinú y San Jorge-Bajo Cauca Antioqueño": "guarida" del paludismo colombiano. Rev Univ Ind Santander 2017; 49: 12. https://revistas.uis. edu.co/index.php/revistasaluduis/article/ view/7620/7859.

3.- Arango E M, Samuel R, Agudelo O M, Carmona-Fonseca J, Maestre A, Yanow S K. Molecular detection of malaria at delivery reveals a high frequency of submicroscopic infections and associated placental damage in pregnant women from northwest Colombia. Am J Trop Med Hyg 2013; 89: 178-83. doi: 10.4269/ajtmh.12-0669.

4.- Agudelo O M, Aristizabal B H, Yanow S K, Arango E, Carmona-Fonseca J, Maestre A. Submicroscopic infection of placenta by Plasmodium produces Th1/Th2 cytokine imbalance, inflammation and hypoxia in women from north-west Colombia. Malar J 2014; 13: 122. doi: 10.1186/1475-2875-13-122.
5.- Piñeros-Jiménez J G, Álvarez G, Tobón A, Arboleda M, Carrero S, Blair S. Congenital malaria in Urabá, Colombia. Malar J 2011; 10 : 239. doi: 10.1186/1475-2875-10-239.

6.- Carmona-Fonseca J, Arango E, Maestre A. Placental malaria in Colombia: histopathologic findings in Plasmodium vivax and $P$. falciparum infections. Am J Trop Med Hyg 2013; 88: 1093-101. doi: 10.4269/ajtmh.12-0363.

7.- Ismaili J, van der Sande M, Holland M J, Sambou I, Keita S, Allsopp C, et al. Plasmodium falciparum infection of the placenta affects newborn immune responses. Clin Exp Immunol 2003; 133: 414-21. PMCID: PMC180879.

8.- Le Port A, Watier L, Cottrell G, Ouédraogo S, Dechavanne $\mathrm{C}$, Pierrat $\mathrm{C}$, et al. Infections in infants during the first 12 months of life: role of placental malaria and environmental factors. PLoS One 2011; 6:e27516. doi: 10.1371/ journal.pone.0027516.

9.- Dauby N, Goetghebuer T, Kollmann T R, Levy J, Marchant A. Uninfected but not unaffected: chronic maternal infections during pregnancy, fetal immunity, and susceptibility to postnatal infections. Lancet Infect Dis 2012; 12: 330-40. doi: 10.1016/S1473-3099(11)70341-3.

10.- Malhotra I, Dent A, Mungai P, Wamachi A, Ouma J H, Narum D L, et al. Can prenatal malaria exposure produce an immune tolerant phenotype? A prospective birth cohort study in Kenya. PLoS Med 2009; 6:e1000116. doi: 10.1371/journal.pmed.1000116.

11.- Labeaud A D, Malhotra I, King M J, King C L, King C H. Do antenatal parasite infections devalue childhood vaccination? PLoS Negl Trop Dis 2009; 3:e442. doi: 10.1371/journal. pntd.0000442.

12.- Dietz V, Galazka A, van Loon F, Cochi S. Factors affecting the immunogenicity and potency of tetanus toxoid: implications for the elimination of neonatal and nonneonatal tetanus as public health problems. Bull World Health Organ 1997; 75: 81-93. PMCID: PMC2486988.

13.- Usen S, Milligan P, Ethevenaux C, Greenwood B, Mulholland K. Effect of fever on the serum antibody response of Gambian children to Haemophilus influenzae type b conjugate vaccine. Pediatr Infect Dis J 2000; 19: 444-9. PMID: 10819341.

14.- Walther B, Miles D J, Waight P, Palmero M S, Ojuola O, Touray E S, et al. Placental malaria is associated with attenuated CD4 T-cell responses to tuberculin PPD 12 months after BCG vaccination. BMC Infect Dis 2012; 12: 6 . doi: 10.1186/1471-2334-12-6.

15.- Gyhrs A, Pedersen B K, Bygbjerg I, Henrichsen J, Heron I, Petersen I, et al. The effect of prophylaxis with chloroquine and proguanil 
on delayed-type hypersensitivity and antibody production following vaccination with diphtheria, tetanus, polio, and pneumococcal vaccines. Am J Trop Med Hyg 1991; 45: 613-8. PMID: 1951872.

16.- Cumberland P, Shulman C E, Maple P A, Bulmer J N, Dorman E K, Kawuondo K, et al. Maternal HIV infection and placental malaria reduce transplacental antibody transfer and tetanus antibody levels in newborns in Kenya. J Infect Dis 2007; 196: 550-7. doi: $10.1086 / 519845$.

17.- Álvarez-Larrotta C, Agudelo O M, Duque Y, Gavina K, Yanow S K, Maestre A, et al. Submicroscopic Plasmodium infection during pregnancy is associated with reduced antibody levels to tetanus toxoid. Clin Exp Immunol 2019; 195 (1): 96-108. doi: 10.1111/cei.13213.

18.- Frosch A E, John C C. Immunomodulation in Plasmodium falciparum malaria: experiments in nature and their conflicting implications for potential therapeutic agents. Expert Rev Anti Infect Ther 2012; 10: 1343-56. doi: 10.1586/ eri.12.118.

19.- Gavina K, Gnidehou S, Arango E, HamelMartineau C, Mitran C, Agudelo O, et al. Clinical outcomes of submicroscopic infections and correlates of protection of VAR2CSA antibodies in a longitudinal study of pregnant women in Colombia. Infect Immun 2018; 86 (4). pii: e00797-17. doi: 10.1128/IAI.00797-17.

20.- Agudelo-García O M, Arango-Flórez E M, Carmona-Fonseca J. Submicroscopic and asymptomatic congenital infection by Plasmodium vivax or $P$. falciparum in Colombia: 37 cases with placental histopathology and cytokine profile in maternal and placental blood. J Trop Med 2017; 2017: 3680758. doi: $10.1155 / 2017 / 3680758$.

21.- Xie J, Tato C M, Davis M M. How the immune system talks to itself: the varied role of synapses. Immunol Rev 2013; 251: 65-79. doi: 10.1111/imr.12017.

22.- Davis D M. Intrigue at the immune synapse. Sci Am 2006; 294 (2): 448-55. PMID: 16478026.

23.- Couper K N, Blount D G, Wilson M S, Hafalla J C, Belkaid Y, Kamanaka M, et al. IL-10 from CD4CD25Foxp3CD127 adaptive regulatory $\mathrm{T}$ cells modulates parasite clearance and pathology during malaria infection. PLoS Pathog 2008; 4:e1000004. doi: 10.1371/journal. ppat. 1000004.

24.- Minigo G, Woodberry T, Piera K A, Salwati E, Tjitra E, Kenangalem E, et al. Parasitedependent expansion of TNF receptor II-positive regulatory $\mathrm{T}$ cells with enhanced suppressive activity in adults with severe malaria. PLoS Pathog 2009; 5:e1000402. doi: 10.1371/journal.ppat.1000402.

25.- Mota M M, Rodríguez A. Invasion of mammalian host cells by Plasmodium sporozoites. Bioessays 2002; 24: 149-56. doi: 10.1002/bies.10050.

26.- Nussler A K, Rénia L, Pasquetto V, Miltgen $\mathrm{F}$, Matile H, Mazier D. In vivo induction of the nitric oxide pathway in hepatocytes after injection with irradiated malaria sporozoites, malaria blood parasites or adjuvants. Eur J Immunol 1993; 23: 882-7. doi: 10.1002/ eji.1830230417.

27.- Seguin M C, Klotz F W, Schneider I, Weir J $\mathrm{P}$, Goodbary M, Slayter M, et al. Induction of nitric oxide synthase protects against malaria in mice exposed to irradiated Plasmodium berghei infected mosquitoes: involvement of interferon gamma and CD8+ T cells. J Exp Med 1994; 180: 353-8. PMCID: PMC2191552.

28.- Wipasa J, Suphavilai C, Okell L C, Cook J, Corran P H, Thaikla K, et al. Long-lived antibody and $\mathrm{B}$ cell memory responses to the human malaria parasites, Plasmodium falciparum and Plasmodium vivax. PLoS Pathog 2010; 6:e1000770. doi: 10.1371/journal. ppat.1000770.

29.- Yoshida N, Nussenzweig R S, Potocnjak P, Nussenzweig V, Aikawa M. Hybridoma produces protective antibodies directed against the sporozoite stage of malaria parasite. Science 1980; 207: 71-3. PMID: 6985745.

30.- Potocnjak P, Yoshida N, Nussenzweig R S, Nussenzweig V. Monovalent fragments (Fab) of monoclonal antibodies to a sporozoite surface antigen $(\mathrm{Pb} 44)$ protect mice against malarial infection. J Exp Med 1980; 151: 1504-13. PMID: 6991628

31.- Plebanski M, Hannan C M, Behboudi S, Flanagan K L, Apostolopoulos V, Sinden R $\mathrm{E}$, et al. Direct processing and presentation of antigen from malaria sporozoites by professional antigen-presenting cells in the induction of CD8 T-cell responses. Immunol Cell Biol 2005; 83: 307-12. doi: 10.1111/j.1440-1711.2005.01325.x

32.- Wilson N S, Villadangos J A. Regulation of antigen presentation and cross-presentation in the dendritic cell network: facts, hypothesis, and immunological implications. Adv Immunol 2005; 86: 241-305. doi: 10.1016/S00652776(04)86007-3.

33.- Krishnegowda G, Hajjar A M, Zhu J, Douglass E J, Uematsu S, Akira S, et al. Induction of proinflammatory responses in macrophages by the glycosylphosphatidylinositols of Plasmodium falciparum: cell signaling receptors, glycosylphosphatidylinositol (GPI) structural requirement, and regulation of GPI activity. J Biol Chem 2005; 280: 8606-16. doi: 10.1074/jbc.M413541200.

34.- Kalantari P, De Oliveira R B, Chan J, Corbett Y, Rathinam V, Stutz A, et al. Dual engagement of the NLRP3 and AIM2 inflammasomes by plasmodium-derived hemozoin and DNA during malaria. Cell Rep 2014; 6: 196-210. doi: 10.1016/j.celrep.2013.12.014.
35.- Yarovinsky F, Zhang D, Andersen J F, Bannenberg G L, Serhan C N, Hayden M S, et al. TLR11 activation of dendritic cells by a protozoan profilin-like protein. Science 2005 ; 308: 1626-9. doi: 10.1126/science.1109893.

36.- Baratin M, Roetynck S, Lépolard C, Falk C, Sawadogo S, Uematsu S, et al. Natural killer cell and macrophage cooperation in MyD88dependent innate responses to Plasmodium falciparum. Proc Natl Acad Sci USA 2005; 102: 14747-52. doi: 10.1073/pnas.0507355102.

37.- Kinyanjui S M. Malaria Parasites. In: Omolade $\mathrm{O}$, ed. The immunology of malaria. InTech, 2012. doi 10.5772/34826.

38.- Legorreta-Herrera M, Rivas-Contreras S, Ventura-Gallegos J, Zentella-Dehesa A. Nitric oxide is involved in the upregulation of IFN- $\gamma$ and IL-10 mRNA expression by $\mathrm{CD} 8^{+} \mathrm{T}$ cells during the blood stages of $P$. chabaudi AS infection in CBA/Ca mice. Int J Biol Sci 2011; 7: 1401-11. PMCID: PMC3221947.

39.- Namazi M J, Phillips R S. Immune responses of $\mathrm{NIH}$ mice infected with avirulent and virulent strains of Plasmodium chabaudi adami single and mixed infections. Korean J Parasitol 2010; 48: 23-33. doi: 10.3347/kjp.2010.48.1.23.

40.- Tongren J E, Drakeley C J, McDonald S L, Reyburn H G, Manjurano A, Nkya W M, et al. Target antigen, age, and duration of antigen exposure independently regulate immunoglobulin $\mathrm{G}$ subclass switching in malaria. Infect Immun 2006; 74: 257-64. doi: 10.1128/IAI.74.1.257-264.2006.

41.- Stanisic D I, Richards J S, McCallum F J, Michon P, King C L, Schoepflin S, et al. Immunoglobulin $\mathrm{G}$ subclass-specific responses against Plasmodium falciparum merozoite antigens are associated with control of parasitemia and protection from symptomatic illness. Infect Immun 2009; 77: 1165-74. doi: 10.1128/IAI.01129-08.

42.- Chua C L, Brown G, Hamilton J A, Rogerson $\mathrm{S}$, Boeuf P. Monocytes and macrophages in malaria: protection or pathology? Trends Parasitol 2013; 29: 26-34. doi: 10.1016/j. pt.2012.10.002.

43.- Flick K, Chen Q. var genes, PfEMP1 and the human host. Mol Biochem Parasitol 2004; 134 : 3-9. PMID: 14747137.

44.- Gowda N M, Wu X, Kumar S, Febbraio M, Gowda D C. CD36 contributes to malaria parasite-induced pro-inflammatory cytokine production and $\mathrm{NK}$ and $\mathrm{T}$ cell activation by dendritic cells. PLoS One 2013; 8:e77604. https://doi.org/10.1371/journal.pone.0077604.

45.- Smith J D, Rowe J A, Higgins M K, Lavstsen T. Malaria's deadly grip: cytoadhesion of Plasmodium falciparum-infected erythrocytes. Cell Microbiol 2013; 15: 1976-83. doi: 10.1111/ cmi.12183.

46.- Deshmukh A S, Srivastava S, Dhar S K Plasmodium falciparum: epigenetic control 
of var gene regulation and disease. Subcell Biochem 2013; 61: 659-82. doi: 10.1007/97894-007-4525-4 28.

47.- Schwarzer E, Arese P. Phagocytosis of malarial pigment hemozoin inhibits NADPHoxidase activity in human monocyte-derived macrophages. Biochim Biophys Acta 1996; 1316: 169-75. PMID: 8781535 .

48.- Berkley J, Mwarumba S, Bramham K, Lowe B, Marsh K. Bacteraemia complicating severe malaria in children. Trans R Soc Trop Med Hyg 1999; 93: 283-6. PMID: 10492760.

49.- Church J, Maitland K. Invasive bacterial coinfection in African children with Plasmodium falciparum malaria: a systematic review. BMC Med 2014; 12: 31. doi: 10.1186/1741-7015-1231.

50.- Good M F. Malaria. Tying the conductor's arms. Nature 1999; 400: (6739): 25-6. doi: $10.1038 / 21793$

51.- Wykes M N, Kay J G, Manderson A, Liu X Q, Brown D L, Richard D J, et al. Rodent blood-stage Plasmodium survive in dendritic cells that infect naive mice. Proc Natl Acad Sci U S A 2011; 108: 11205-10. doi: 10.1073/ pnas. 1108579108.

52.- Wykes M N, Horne-Debets J. Dendritic cells: the Trojan horse of malaria? Int J Parasitol 2012; 42: 583-7. doi: 10.1016/j. ijpara.2012.03.002.

53.- Arama C, Troye-Blomberg M. The path of malaria vaccine development: challenges and perspectives. J Intern Med 2014; 275: 456-66. doi: 10.1111/joim.12223.

54.- Li C, Corraliza I, Langhorne J. A defect in interleukin-10 leads to enhanced malarial disease in Plasmodium chabaudi chabaudi infection in mice. Infect Immun 1999; 67: 4435-42. PMCID: PMC96762.

55.- Luckheeram R V, Zhou R, Verma A D, Xia B. $C D 4^{+} T$ cells: differentiation and functions. Clin Dev Immunol 2012; 2012: 925135. doi: 10.1155/2012/925135.

56.- Thornton A M, Shevach E M. CD4+CD25+ immunoregulatory $\mathrm{T}$ cells suppress polyclonal $\mathrm{T}$ cell activation in vitro by inhibiting interleukin 2 production. J Exp Med 1998; 188: 287-96. PMCID: PMC2212461.

57.- Bluestone J A, Abbas A K. Natural versus adaptive regulatory $\mathrm{T}$ cells. Nat Rev Immunol 2003; 3: 253-7. doi: 10.1038/nri1032.

58.- Walther M, Jeffries D, Finney O C, Njie M, Ebonyi A, Deininger S, et al. Distinct roles for FOXP3 and FOXP3 CD4 T cells in regulating cellular immunity to uncomplicated and severe Plasmodium falciparum malaria. PLoS Pathog 2009; 5:e1000364. doi: 10.1371/journal. ppat. 1000364.

59.- Scholzen A, Minigo G, Plebanski M. Heroes or villains? T regulatory cells in malaria infection. Trends Parasitol 2010; 26: 16-25. doi: 10.1016/j.pt.2009.10.004
60.- Wammes L J, Wiria A E, Toenhake C G, Hamid F, Liu K Y, Suryani H, et al. Asymptomatic plasmodial infection is associated with increased tumor necrosis factor receptor IIexpressing regulatory $\mathrm{T}$ cells and suppressed type 2 immune responses. J Infect Dis 2013; 207: 1590-9. doi: 10.1093/infdis/jit058.

61.- Hisaeda H, Tetsutani K, Imai T, Moriya C, Tu L, Hamano S, et al. Malaria parasites require TLR9 signaling for immune evasion by activating regulatory T cells. J Immunol 2008; 180: 2496-503. PMID: 18250459.

62.- Barteneva N S, Maltsev N, Vorobjev I A. Microvesicles and intercellular communication in the context of parasitism. Front Cell Infect Microbiol 2013; 3: 49. doi: 10.3389/ fcimb.2013.00049.

63.- Couper K N, Barnes T, Hafalla J C, Combes V, Ryffel B, Secher T, et al. Parasite-derived plasma microparticles contribute significantly to malaria infection-induced inflammation through potent macrophage stimulation. PLoS Pathog 2010; 6:e1000744. doi: 10.1371/journal. ppat.1000744.

64.- Regev-Rudzki N, Wilson D W, Carvalho T G, Sisquella X, Coleman B M, Rug M, et al. Cellcell communication between malaria-infected red blood cells via exosome-like vesicles. Cell 2013; 153: 1120-33. doi: 10.1016/j. cell.2013.04.029.

65.- Inal J M, Kosgodage U, Azam S, Stratton D, Antwi-Baffour S, Lange S. Blood/plasma secretome and microvesicles. Biochim Biophys Acta 2013; 1834: 2317-25. doi: 10.1016/j. bbapap.2013.04.005.

66.- LaMonte G, Philip N, Reardon J, Lacsina J R, Majoros W, Chapman L, et al. Translocation of sickle cell erythrocyte microRNAs into Plasmodium falciparum inhibits parasite translation and contributes to malaria resistance. Cell Host Microbe 2012; 12: 187-99. doi: 10.1016/j.chom.2012.06.007.

67.- Del Portillo H A, Chitnis C E. Talking to each other to initiate sexual differentiation. Cell 2013; 153: 945-7. doi: 10.1016/j. cell.2013.05.013

68.- Campos F M, Franklin B S, Teixeira-Carvalho A, Filho A L, de Paula S C, Fontes C J, et al. Augmented plasma microparticles during acute Plasmodium vivax infection. Malar J 2010; 9: 327. doi: 10.1186/1475-2875-9-327.

69.- Mantel P Y, Schmidt-Weber C B. Transforming growth factor-beta: recent advances on its role in immune tolerance. Methods Mol Biol 2011; 677: 303-38. doi: 10.1007/978-1-60761-869$0 \_21$.

70.- Pombo D, Maloy W L, Berzofsky J A, Good M F. Neonatal exposure to immunogenic peptides. Differential susceptibility to tolerance induction of helper T cells and B cells reactive to malarial circumsporozoite peptide epitopes. J Immunol 1988; 140: 3594-8. PMID 2452193.
71.- Harte P G, Playfair J H. Failure of malaria vaccination in mice born to immune mothers. II. Induction of specific suppressor cells by maternal IgG. Clin Exp Immunol 1983; 51: 157-64. PMCID: PMC1536748.

72.- Murray C J, Ortblad K F, Guinovart C, Lim S S, Wolock T M, Roberts D A, et al. Global, regional, and national incidence and mortality for HIV, tuberculosis, and malaria during 1990-2013: a systematic analysis for the Global Burden of Disease Study 2013. Lancet 2014; 384: 1005-70. doi: 10.1016/S01406736(14)60844-8.

73.- Ibitokou S, Oesterholt M, Brutus L, Borgella $\mathrm{S}$, Agbowaï C, Ezinmègnon S, et al. Peripheral blood cell signatures of Plasmodium falciparum infection during pregnancy. PLoS One 2012; 7:e49621. doi: 10.1371/journal.pone.0049621.

74.- Le Hesran J Y, Cot M, Personne P, Fievet N, Dubois B, Beyemé M, et al. Maternal placental infection with Plasmodium falciparum and malaria morbidity during the first 2 years of life. Am J Epidemiol 1997; 146: 826-31. PMID: 9384203.

75.- Mutabingwa T K, Bolla M C, Li J L, Domingo G J, Li X, Fried M, et al. Maternal malaria and gravidity interact to modify infant susceptibility to malaria. PLoS Med 2005; 2: 407. doi: 10.1371/journal.pmed.0020407.

76.- Schwarz N G, Adegnika A A, Breitling L P, Gabor J, Agnandji S T, Newman R D, et al. Placental malaria increases malaria risk in the first 30 months of life. Clin Infect Dis 2008; 47: 1017-25. doi: 10.1086/591968.

77.- Cairns M, Gosling R, Chandramohan D. Placental malaria increases malaria risk in the first 30 months of life: not causal. Clin Infect Dis 2009; 48: 497-8; author reply 498-9. doi: $10.1086 / 596548$

78.- Brair M E, Brabin B J, Milligan P, Maxwell S, Hart C A. Reduced transfer of tetanus antibodies with placental malaria. Lancet 1994; 343: 208-9. PMID: 7904669

79.- Soriano-Gabarró M, Toé L, Tiendrebeogo S R, Nelson C B, Dabal M, Djingarey M H, et al; Group WTVIAS. Effectiveness of a trivalent serogroup A/C/W135 meningococcal polysaccharide vaccine in Burkina Faso, 2003. Vaccine 2007; 25 Suppl 1: A92-96. doi: 10.1016/j.vaccine.2007.04.048.

80.- Flanagan K L, Halliday A, Burl S, Landgraf K, Jagne Y J, Noho-Konteh F, et al. The effect of placental malaria infection on cord blood and maternal immunoregulatory responses at birth. Eur J Immunol 2010; 40: 1062-72. doi: 10.1002/eji.200939638.

81.- Lalor M K, Ben-Smith A, Gorak-Stolinska P, Weir R E, Floyd S, Blitz R, et al. Population differences in immune responses to Bacille Calmette-Guérin vaccination in infancy. J Infect Dis 2009; 199: 795-800. doi: 10.1093/infdis/ jir515 